\title{
DATA ANALYSIS USING RAPIDMINER
}

\author{
Masoud Karimi \\ Department of Computer Engineering, \\ University of Kurdistan, Iran \\ m.karimi@eng.uok.ac.ir
}

\section{ABSTRACT}

In this technical report, I have downloaded RapidMiner Studio and an open dataset from data.gov about demographic distribution in New York. I will show some analysis by means of charts provided by RapidMiner.

\section{KEYWORDS}

demographic distribution, data analysis

\section{INTRODUCTION}

Firstly we need to download RapidMiner Studio from the website and create student account. I used the data.gov open data to analyze. From the https://catalog.data.gov/dataset I have downloaded the "Demographic Statistics By Zip Code" csv dataset. The dataset is City of demographic statistics broken down by zip code in New York. Then in RapidMiner I selected the Add Data button and select from my computer the csv file I just downloaded. Following screenshot shows the data that has been loaded.

There are some previous works that have used RapidMiner for data mining such as [1-3]. In the era of big data which there are lots of devices and social networks that produce terabytes of data[4] we need an advanced tools to run data mining tasks. 


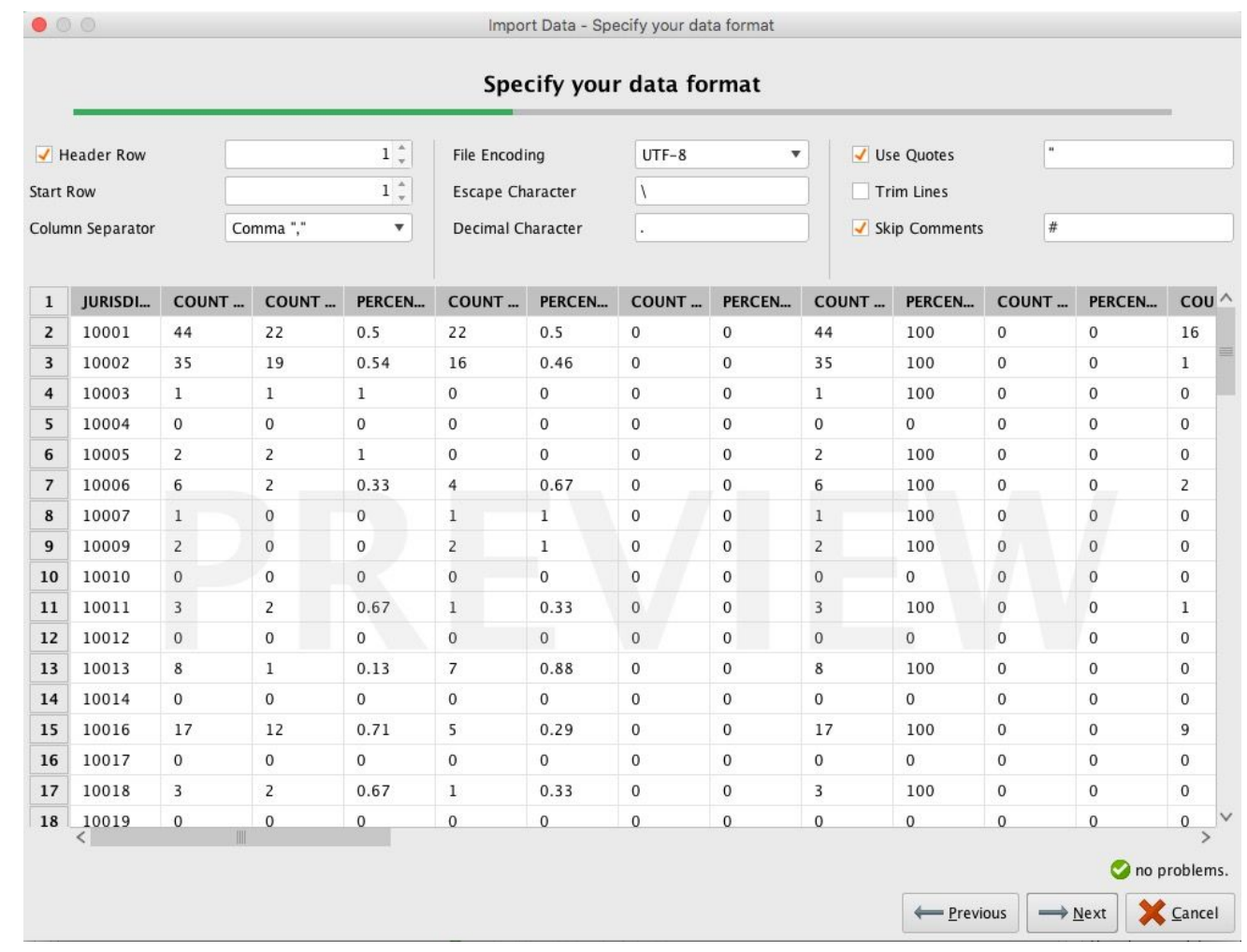

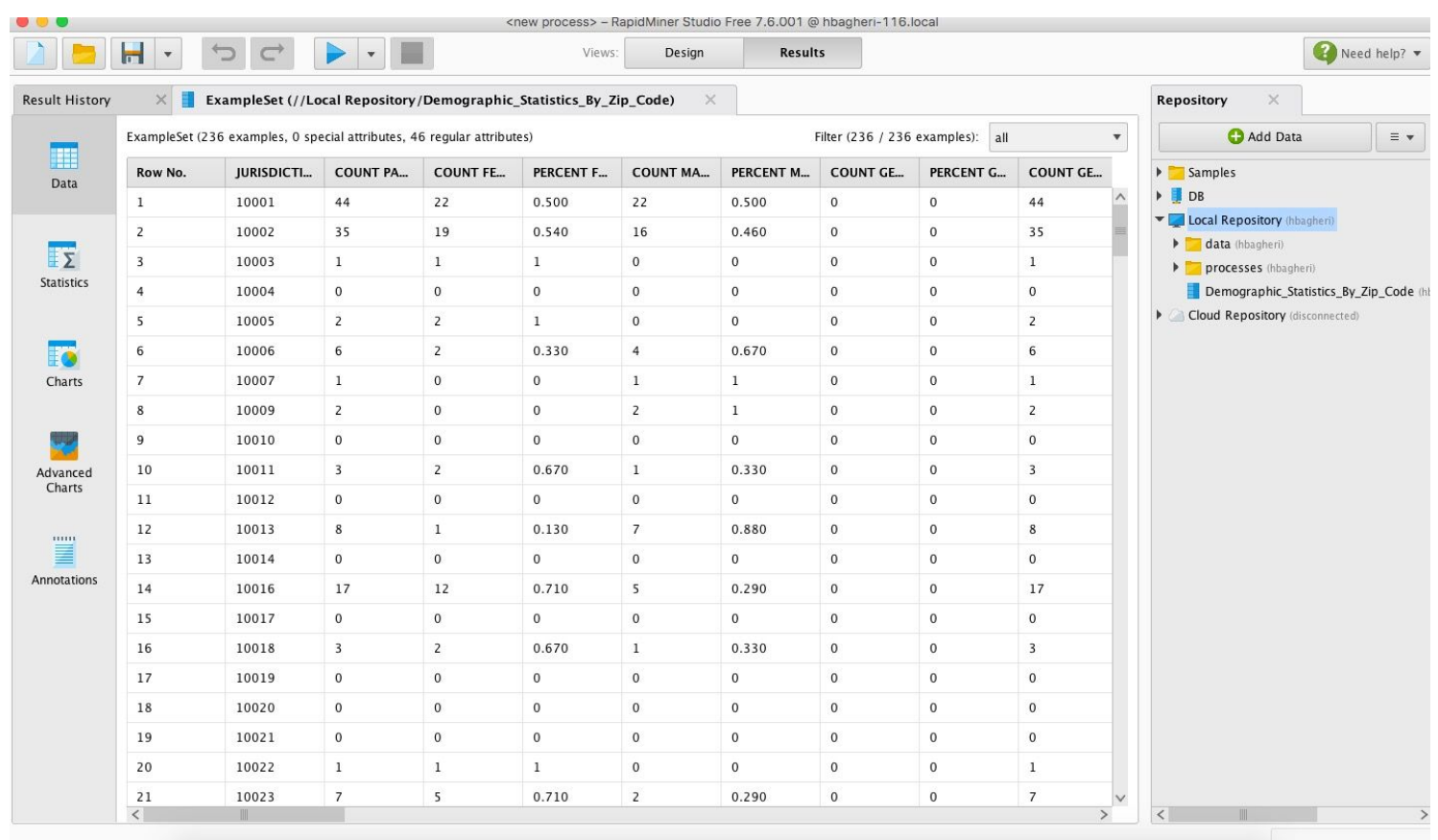




\section{Charts}

From the dataset we have downloaded we can double click and go to the chart tab. There are different plot we can choose for analyze the data. Following is the frequency based on each jurisdiction name (which is basically an ID).

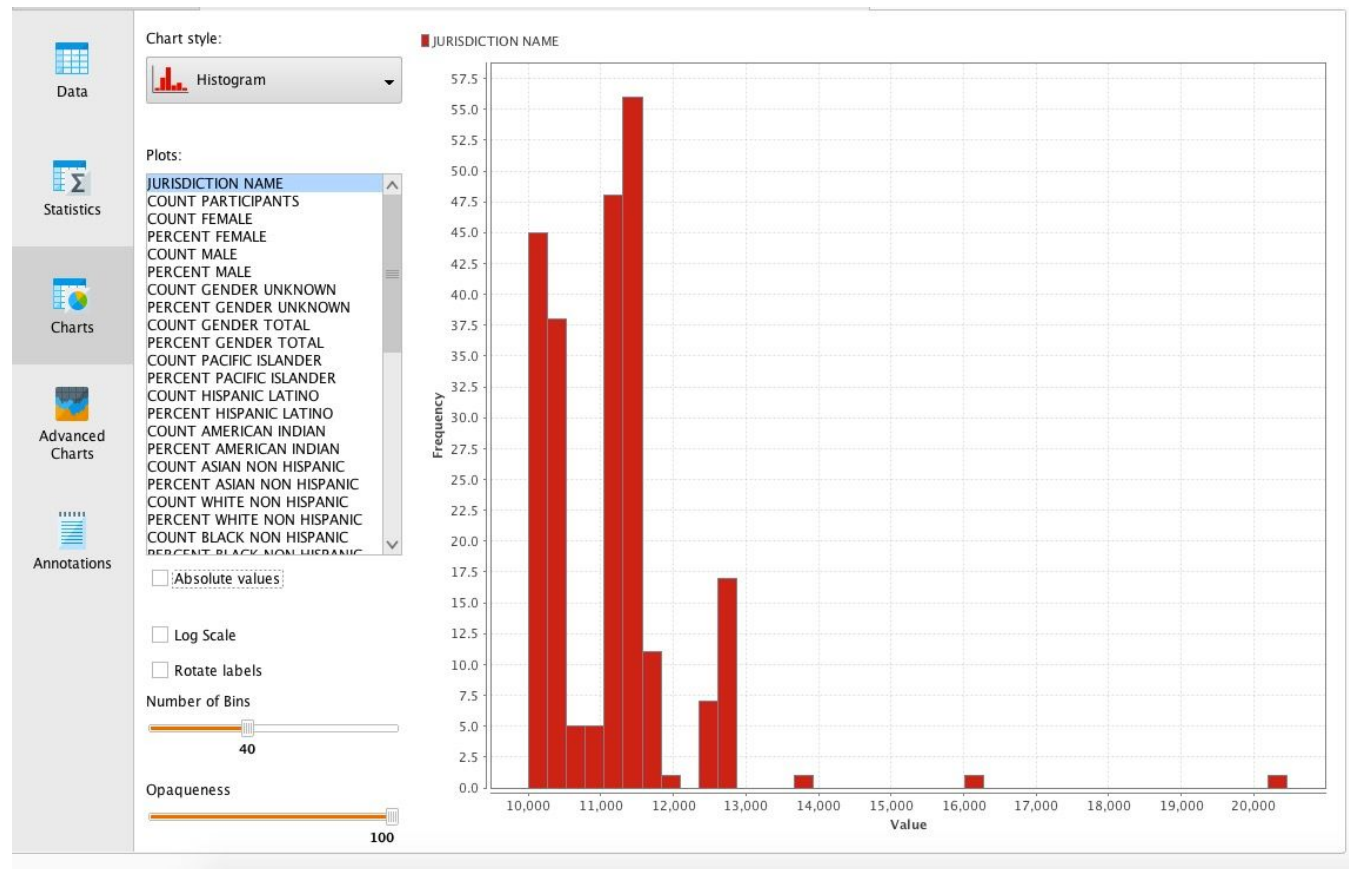

The second plot is scatter plot and here we can change the x-axis and y-axis. In this analysis I selected the Percent of Female in each jurisdiction as an $\mathrm{x}$-Axis and the $\mathrm{y}$-Axis is the percent of the Hispanic and Latino ethnicity group. The purpose of this chart is the Hispanic and Latino distribution based on the Female population. 

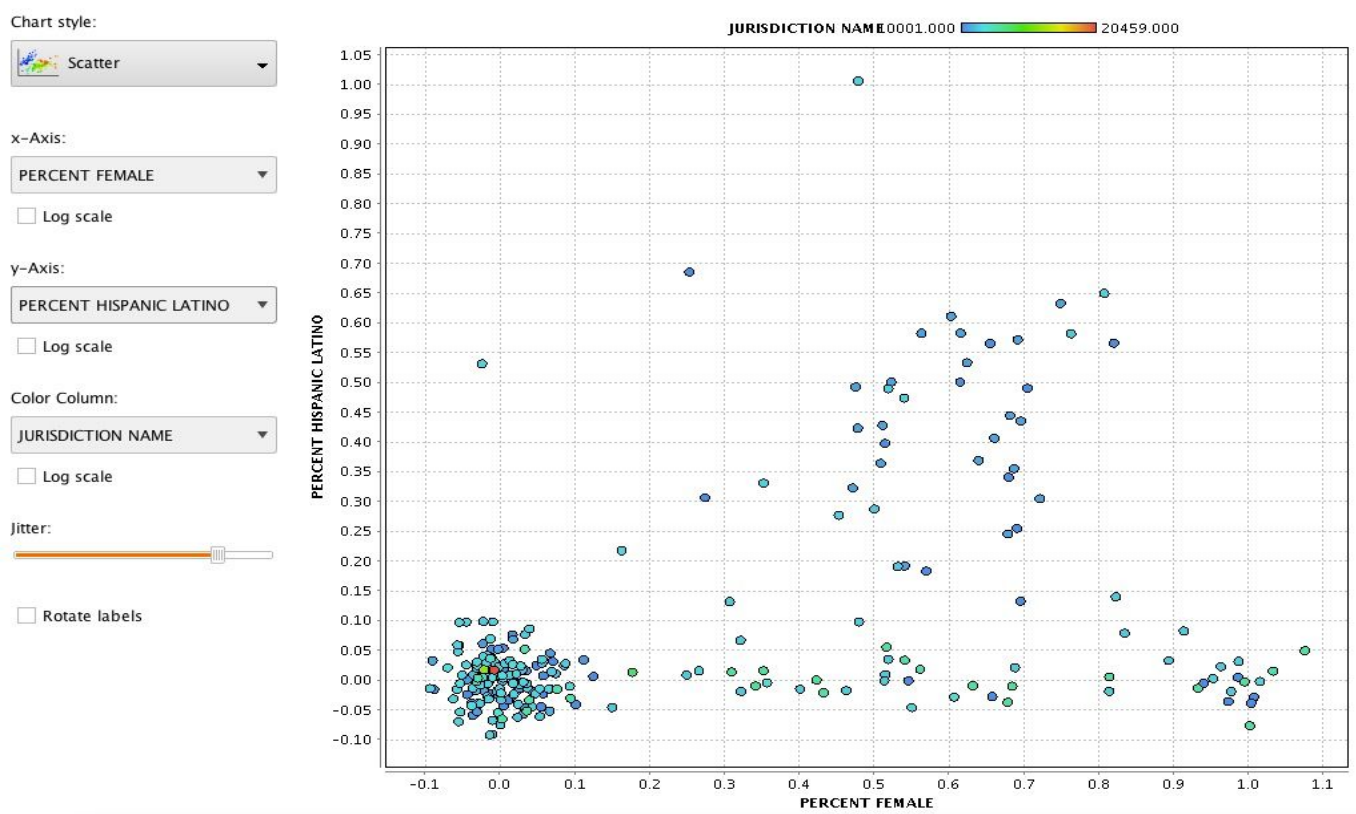

\section{Conclusions}

In this technical report, I have downloaded an open dataset from data.gov about demographic distribution in New York. I have analyzed the data and figured out some insight from data.

\section{REFERENCES}

[1] Hofmann, M., \& Klinkenberg, R. (Eds.). (2013). RapidMiner: Data mining use cases and business analytics applications. CRC Press.

[2] Hall, M., Frank, E., Holmes, G., Pfahringer, B., Reutemann, P., \& Witten, I. H. (2009). The WEKA data mining software: an update. ACM SIGKDD explorations newsletter, 11(1), 10-18.

[3] Ristoski, P., Bizer, C., \& Paulheim, H. (2015). Mining the web of linked data with rapidminer. Web Semantics: Science, Services and Agents on the World Wide Web, 35, 142-151.

[4] Bagheri, H., \& Shaltooki, A. A. (2015). Big Data: challenges, opportunities and Cloud based solutions. International Journal of Electrical and Computer Engineering, 5(2), 340.

[5] Kosorus, H., Honigl, J., \& Kung, J. (2011, August). Using R, WEKA and RapidMiner in time series analysis of sensor data for structural health monitoring. In Database and Expert Systems Applications (DEXA), 2011 22nd International Workshop on (pp. 306-310). IEEE.

[6] https://catalog.data.gov/dataset

[7] https://my.rapidminer.com/nexus/account/index.html\#downloads 